\title{
Time-resolved luminescence spectroscopy by the optical Kerr-gate method applicable to ultrafast relaxation processes
}

\author{
J. Takeda, K. Nakajima, and S. Kurita \\ Department of Physics, Faculty of Engineering, Yokohama National University, Hodogaya Yokohama 240-8501, Japan \\ S. Tomimoto, S. Saito, and T. Suemoto \\ Institute for Solid State Physics, University of Tokyo, Kashiwa, Chiba 277-8581, Japan
}

(Received 15 May 2000)

\begin{abstract}
We have developed a femtosecond time-resolved luminescence spectroscopy by the optical Kerr-gate (OKG) method to investigate ultrafast carrier dynamics and relaxation processes of materials. Solid glasses with a high nonlinear refractive index were used as the Kerr media to obtain a subpicosecond time resolution. When a quartz plate was used as the Kerr medium, the Kerr efficiency and the instrumental response time of our spectroscopic system were 5-10\% and $\sim 250$ fs, respectively. By employing the OKG method, we revealed the internal conversion from $S_{2}$ to $S_{1}$ state of $\beta$-carotene with a low fluorescence quantum yield and an ultrafast fluorescence decay time, and the lifetime of the $S_{2}$ state was determined to be $210 \mathrm{fs}$. An advantage of the OKG method relative to the conventional up-conversion technique is its ability to directly obtain time-resolved luminescence spectra, and thus the OKG method might be superior to the up-conversion technique to investigate ultrafast carrier dynamics and relaxation processes of materials.
\end{abstract}

\section{INTRODUCTION}

Observation of the transient behavior of excited states is of great importance and interest in studying carrier dynamics and relaxation processes of materials. To investigate such phenomena, the femtosecond up-conversion luminescence spectroscopy has been utilized for the last 10 years as a powerful spectroscopic tool, since Shah and co-workers developed this technique. ${ }^{1-6}$ Since the wavelength and direction of the sum frequency output are far away from those of the detected luminescence and gating laser pulses, the upconversion technique is basically a background-free detection. Time evolution of luminescence is thus sensitively obtained. In this technique, however, the phase match angle of nonlinear crystal to generate the sum frequency output has to be varied when changing the detected luminescence photon energy. This brings difficulties to correct the relative luminescence intensity for different photon energies and to determine the origin of time axis for the delay of the luminescence. As a result, this technique makes it difficult to obtain time-resolved luminescence spectra, which could provide us important and fruitful information on carrier dynamics and relaxation processes of materials.

On the other hand, time-resolved luminescence spectra can be directly obtained by the optical Kerr-gate (OKG) method. In the OKG method, liquids or solutions such as $\mathrm{CS}_{2}$ are generally used as the Kerr media. Since the time response of the optical Kerr effect for liquids/solutions is governed by rotational relaxation of molecules, the typical time resolution of the OKG method is limited to $1-2$ ps so far. ${ }^{7,8}$ Moreover, the OKG method contains substantial backgrounds coming from the scattered light and luminescence itself which survive against the crossed polarizers. Hulin et al. obtained time resolution as short as $0.5 \mathrm{ps}$ in visible light region by using benzene solution as the Kerr medium. ${ }^{9}$ However, the temporal response near ultraviolet light region $(\sim 400 \mathrm{~nm})$ was broader $(\sim 1 \mathrm{ps})$ due to the large group velocity dispersion between the different spectral components of probe pulses, and the contrast ratio available was small because of the component of molecular rotational relaxation with rather long recovery time in the medium. For the above reasons, it seems difficult to observe the time-resolved luminescence spectra of materials in a wide spectral region with a subpicosecond time resolution by the OKG method.

High refractive solid glasses are known to have a high third-order nonlinear susceptibility and have an instantaneous response because of the absence of the rotational relaxation, ${ }^{10-12}$ implying that they are good candidates as the Kerr media to investigate ultrafast carrier dynamics and relaxation processes of materials. From this point of view, very recently, a few groups tried to measure the time-resolved luminescence spectra of different materials in femtosecond time domain by use of solid glasses as the Kerr media. ${ }^{13-15}$

In this paper, we present a time-resolved luminescence spectroscopy by use of the OKG method with a subpicosecond time resolution using solid glasses as the Kerr media. The Kerr efficiency and the instrumental response time of our spectroscopic system are $5-10 \%$ and $\sim 250 \mathrm{fs}$, respectively, when we use a quartz plate as the Kerr medium. By employing the OKG method, we measured the time-resolved luminescence spectra of $\beta$-carotene with a low quantum fluorescence efficiency and an ultrafast decay time, and the lifetime of the $S_{2}$ state of $\beta$-carotene was determined to be 210 fs. Our results suggest that the femtosecond OKG luminescence spectroscopy is a powerful spectroscopic tool to investigate ultrafast carrier dynamics and relaxation processes of materials, and that the OKG method might replace the upconversion method in the near future. 


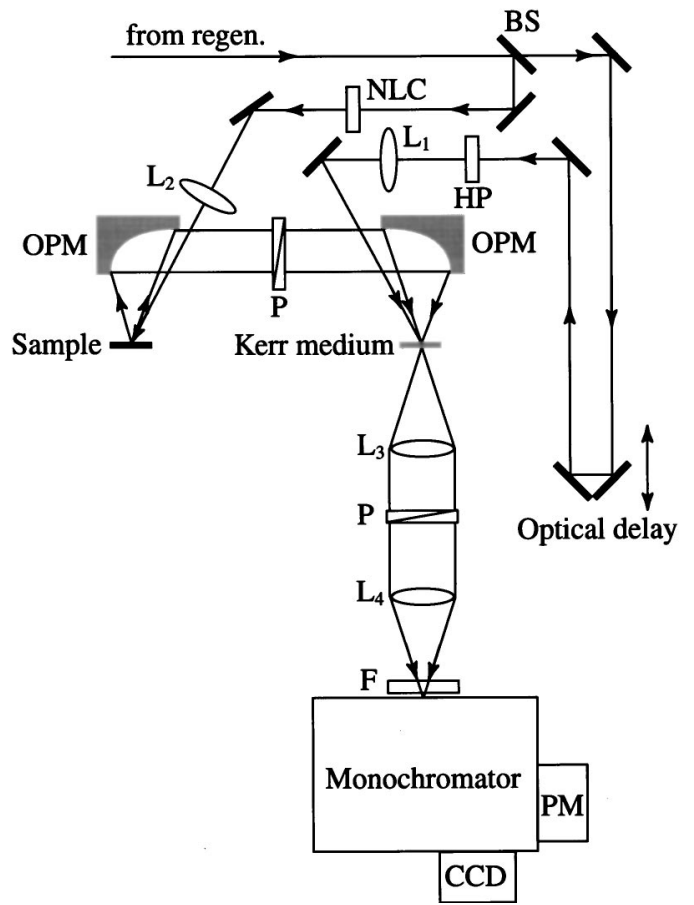

FIG. 1. Experimental setup for the OKG method. BS, beam splitter; NLC, nonlinear crystal (BBO, type $I$ ); $L_{1}, L_{2}$, fused silica lenses; $L_{3}, L_{4}$, achromatic lenses; OPM, off-axis paraboloidal mirror; P, film polarizer; HP, half-wave plate, $F$ interference filter; PM, photomultiplier; $\mathrm{CCD}, \mathrm{CCD}$ detector.

\section{EXPERIMENT}

\section{A. Experimental setup}

Second harmonic $(400 \mathrm{~nm})$ and fundamental laser pulses $(800 \mathrm{~nm})$ from a commercial Ti:sapphire regenerative amplifier laser system (Spectra-Physics, Spitfire) with a 100-fs pulse duration and a $1-\mathrm{kHz}$ repetition rate were used as excitation and gating pulses, respectively. Figure 1 illustrates a schematic diagram of our optical setup. The fundamental beams from the regenerative amplifier were divided into two beams at a beam splitter (BS); one is used as the excitation pulse of sample after the second harmonic generation at a nonlinear crystal (NLC) and the other as the gating laser pulse to rotate the polarization of luminescence at a Kerr medium. The Kerr medium was placed between crossed polarizers $(\mathrm{P})$. To reduce the group velocity dispersion of our optical setup, film polarizers with an extinction ratio of $10^{3}-10^{4}$ in visible light region and off-axis paraboloidal mirrors (OPM) were used. The luminescence or scattered light from a sample was collected by the OPM's. The use of the OPM's instead of lenses eliminates an aberration among different wavelengths of detected luminescence/scattered light at the Kerr medium. The luminescence/scattered light from the sample after passing through the first polarizer was focused into the Kerr medium together with gating pulses, which were variably delayed by a translation stage (Meiritsu, MS-C600) with a minimum step of $1 \mu \mathrm{m}$. The spot sizes of gating pulses and luminescence/scattered light at the Kerr medium were arranged at $\sim 300 \times 300$ and $\sim 200 \times 200 \mu \mathrm{m}^{2}$, respectively. The time-resolved luminescence then passed through the second polarizer and was focused into an entrance slit of a monochromator (Acton, Spectrapro-500) with

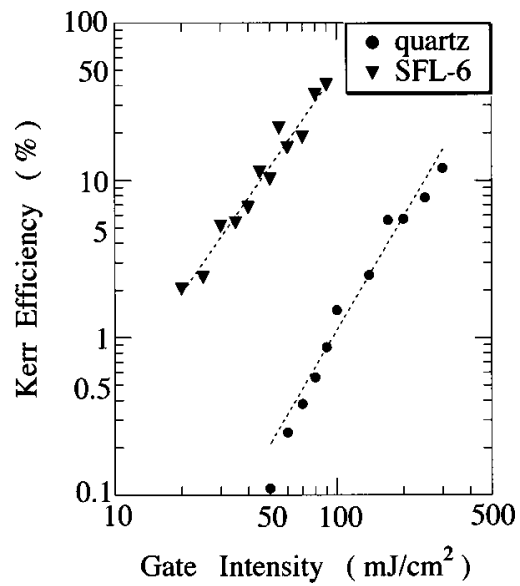

FIG. 2. Log-log plot of the Kerr efficiency of quartz and SFL-6 with a thickness of $0.5 \mathrm{~mm}$ vs gate intensity.

a charge-coupled device (CCD) detector (Princeton Instruments, CCD-1100PB) cooled by liquid nitrogen and a photomultiplier (Hamamatsu, R-928). These detectors can be easily switched on and off by moving an internal mirror of the monochromator. Time-resolved luminescence spectra were detected by the CCD detector, while time evolution of third order correlation function of the scattered excitation pulses was detected by a lock-in amplifier (Stanford Research, SR510).

\section{B. Evaluation of our OKG method}

To obtain a higher Kerr efficiency and a better time resolution, we examined two types of solid glasses with a thickness of $0.25,0.5$, and $1 \mathrm{~mm}$ as the Kerr media; one is a quartz plate and the other is a SFL-6 (SCHOTT GLASS) plate with nonlinear refractive indexes of $1.1 \times 10^{-13}$ and $9.9 \times 10^{-13}$ esu, ${ }^{16}$ respectively. The quartz is transparent above $200 \mathrm{~nm}$, the SFL-6 above $400 \mathrm{~nm}$. Both Kerr media are therefore suited for time-resolved luminescence measurements in the visible light region.

The Kerr efficiency and the overall time resolution of our OKG spectroscopic system were evaluated by the Kerr signal (third order correlation function) of the scattered excitation pulses $(400 \mathrm{~nm})$ from a diffusive glass plate placed at the sample position. The Kerr efficiency is defined by the ratio of intensities of the Kerr signal relative to the total scattered light passed through the polarizers whose polarization direction is parallel.

Figure 2 shows the Kerr efficiency of the quartz and SFL-6 plates with a thickness of $0.5 \mathrm{~mm}$ as a function of gate intensity in a log-log plot. Solid circles and triangles show the observed Kerr efficiency of the quartz and SFL-6, respectively. The Kerr efficiency follows a $x$ th power law of the gate intensity as shown by the broken lines; the value of $x$ for the quartz is 2.4 and that for the SFL-6 is 2.0. For the same gate intensity, the Kerr efficiency of the SFL-6 is 60-80 times larger than that of the quartz. For all the measurements, generation of a white-light continuum or presence of any higher-order nonlinear effect at the Kerr medium was not observed. 


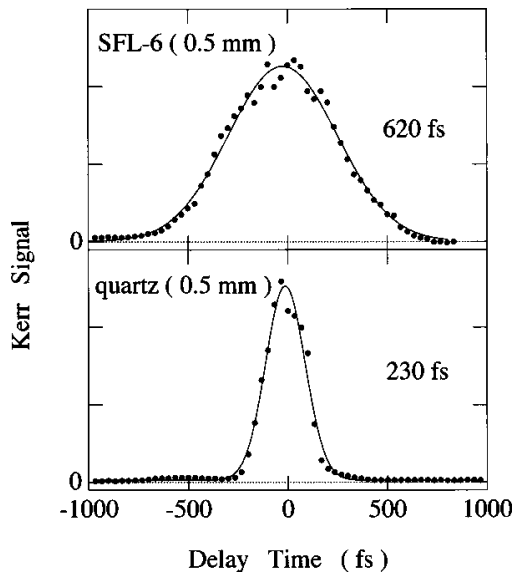

FIG. 3. Time response of third order correlation function of the scattered excitation pulses for quartz and SFL-6 plates. Dots show the experimental data and solid curves indicate the Gaussian fits. The FWHM's of the correlation function for the quartz and SFL-6 are 230 and $620 \mathrm{fs}$, respectively.

A strong electric field by the gating pulses induces an optical anisotropy and consequently a transient birefringence in an isotropic Kerr medium. The refractive index $n$ is given by

$$
n=n_{0}+n_{2}\left\langle E^{2}\right\rangle,
$$

where $n_{0}$ is the linear refractive index, $n_{2}$ the nonlinear refractive index, and $E$ the applied optical electric field. The phase shift $\Phi$ induced by the birefringence depends on the applied optical electric field

$$
\Phi=\frac{2 \pi l}{\lambda} n_{2}\left\langle E^{2}\right\rangle,
$$

where $l$ is the path length traveled in the Kerr medium by the light of a wavelength $\lambda$. The observed transmitted intensity (Kerr signal) $I_{k}$ then depends on the induced phase shift $\Phi$ according to

$$
I_{K}=I_{0} \sin ^{2}(\Phi / 2) \approx I_{0}(\Phi / 2)^{2} \propto n_{2}^{2} I_{G}^{2} .
$$

Here, $I_{G}$ is the gate intensity and proportional to the square of the applied electric field. For small values of $\Phi$, the Kerr signal is proportional to the square of the phase shift $\Phi$, that is, the square of $I_{G}$ and $n_{2} \cdot{ }^{17}$ The larger the values of $n_{2}$, $I_{G}$, and $l$, the stronger the obtained Kerr signal is. As shown in Fig. 2, the observed Kerr efficiency increases almost quadratically with the gate intensity $I_{G}$, as indicated by Eq. (3). Because the nonlinear refractive index of the SFL-6 is nine times larger than that of the quartz, the Kerr efficiency of the SFL-6 is about 80 times higher than that of the quartz for the same gate intensity.

Figure 3 shows third order correlation function of the scattered excitation pulses as a function of delay time for the two kinds of Kerr media, the quartz and SFL-6. The gate intensity was arranged to have the same Kerr efficiency of $5 \%$. The third-order correlation function measured by the $\mathrm{OKG}$ method is defined by

$$
G^{(3)}(\tau)=\int_{-\infty}^{+\infty} I_{P}(t) I_{G}^{2}(t+\tau) d t
$$

Here, $I_{G}(t)$ and $I_{P}(t)$ are the intensities of the strong gating pulse and the weak probing pulse (scattered light), respectively. Assuming that the correlation function $G^{(3)}(\tau)$ has a Gaussian shape, the full width of half maximum (FWHM) of the correlation function is $620 \mathrm{fs}$ for the SFL- 6 and $230 \mathrm{fs}$ for the quartz. The time response function of the quartz is much narrower than that of the SFL-6. The SFL-6 slightly absorbs the scattered excitation pulses in the wavelength region of $400 \mathrm{~nm}$, implying that the group velocity dispersion of the SFL-6 around $400 \mathrm{~nm}$ is large. The blue part of the scattered light propagates slower than the red part of the scattered light through the SFL-6, and consequently the time profile of the Kerr signal becomes broader.

The time response function as well as the Kerr efficiency depends on the thickness of the Kerr medium. The Kerr efficiency increases while increasing the path length of the Kerr medium by Eqs. (2) and (3). On the other hand, the time response function becomes broader while increasing the path length due to the group velocity dispersion of the Kerr medium. In the case of the quartz, for instance, the Kerr efficiency and the time response were $10 \%$ and $350 \mathrm{fs}(1 \mathrm{~mm})$, $5 \%$ and $230 \mathrm{fs}(0.5 \mathrm{~mm})$, and $3 \%$ and $190 \mathrm{fs}(0.25 \mathrm{~mm})$ for the same gate intensity of $200 \mathrm{~mJ} / \mathrm{cm}^{2}$.

We obtained the Kerr efficiency of 5-10\% and the width of the time response function of $\sim 250 \mathrm{fs}$ when we used a quartz plate with a thickness of $0.5 \mathrm{~mm}$ as the Kerr medium. In the up-conversion technique, the efficiency of the sum frequency generation and time resolution of the system are typically $\sim 10 \%$ and $\sim 200 \mathrm{fs}$, respectively, when one uses the fundamental and second harmonic pulses as the gating and the excitation pulses, respectively, from a femtosecond laser system having a pulse duration of $100 \mathrm{fs} .{ }^{4,6}$ We therefore conclude that a quartz plate with a thickness of $0.5 \mathrm{~mm}$ is a reasonable choice as the Kerr medium, and that the obtained Kerr efficiency and time resolution are enough for measurements of ultrafast carrier dynamics and relaxation processes of materials.

\section{EXPERIMENTAL DEMONSTRATIONS}

To demonstrate performance of our OKG method, we measured the time-resolved luminescence spectra of $\beta$-carotene, which has two energetically low-lying singlet states; one is related to the $2^{1} A_{g}\left(S_{1}\right)$ state, which is dipole forbidden from the ground state by the parity conservation, and the other is related to the $1^{1} B_{u}\left(S_{2}\right)$ state, which is responsible for strong absorption in visible light region (Fig. 4). ${ }^{18,19}$ The optical transition to the $S_{2}$ state induces an ultrafast internal conversion to the $S_{1}$ state, followed by relaxation to the ground state. The fluorescence quantum yield is therefore very low $\left(10^{-6}-10^{-4}\right)$ (Refs. 20-22) and the lifetime of the $S_{2}$ state is very short (190-250 fs). ${ }^{22,23}$

Purchased $\beta$-carotene (Wako Pure Chemical Industries, special grade) was used as the sample without further purification. The $\beta$-carotene was dissolved in $n$-hexane solution with a concentration of $2.5 \times 10^{-5} \mathrm{M}$. The solution was kept under $\mathrm{N}_{2}$ saturated conditions and circulated by a peristaltic pump through a flow cell with a 1-mm path length during the time-resolved fluorescence measurements.

For the time-resolved fluorescence measurements, the second harmonic $(400 \mathrm{~nm})$ and the fundamental $(800 \mathrm{~nm})$ 


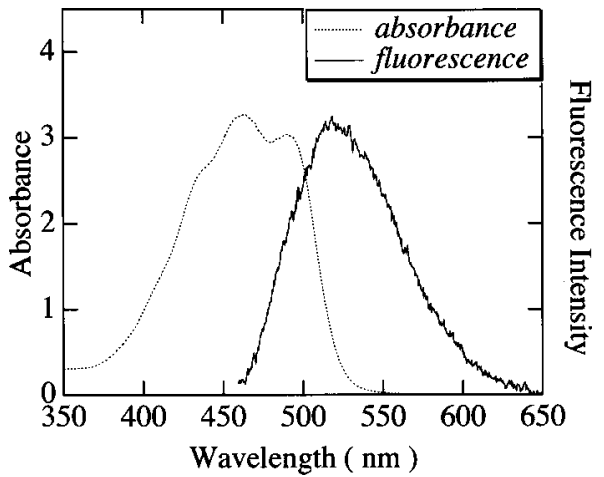

FIG. 4. Absorption (broken curve) and time-integrated fluorescence (solid curve) spectra of $\beta$-carotene in $n$-hexane solution at room temperature.

pulses of the Ti:sapphire regenerative amplifier were used as the excitation pulses of a sample and the gating pulses, respectively. The origin of time axis $(t=0)$ is defined by the time when the intensity of the scattered excitation pulse becomes maximum. The time-resolved fluorescence spectra were measured every 33 fs from -1000 to 1700 fs. The typical accumulation time of the CCD detector to take a time-resolved fluorescence spectrum was 1-2 min. The group velocity dispersion of our optical setup was evaluated by using a white-light continuum generated at $n$-hexane/ water in a flow cell which was placed at the sample position instead of the sample. The chirp of the time-resolved fluorescence spectrum was then corrected by a software procedure on a microcomputer. When the time-integrated fluorescence spectrum was measured, two pieces of the polarizers were set to have a parallel polarization and the gating pulses were blocked.

Figure 4 shows absorption and time-integrated fluorescence spectra of $\beta$-carotene in $n$-hexane solution at room temperature. The strong absorption from the $S_{0}$ to $S_{2}$ state is observed at a visible light region. The broad fluorescence band is located at $\sim 515 \mathrm{~nm}$. The characteristics of these spectra are consistent with those previously reported. ${ }^{22,23}$

Figure 5 shows time-resolved fluorescence spectra of $\beta$-carotene for different delay times at room temperature. The solid curves are added as visual guides to help clarify the peak position and the FWHM of the spectra. The fluorescence spectrum at $t=0$ has a shoulder at $\sim 460 \mathrm{~nm}$, which deviates from the solid curve as shown by a solid arrow. This comes from the scattered light of the excitation pulse. The peak position $(\sim 515 \mathrm{~nm})$ and the FWHM of the spectra $(\sim 80 \mathrm{~nm})$ are almost independent of the delay time. Moreover, these values are in good agreement with those obtained from the time-integrated fluorescence spectrum (Fig. 4). This indicates that the observed time-resolved fluorescence of $\beta$-carotene comes from a single origin. The absence of the dynamic Stokes shift suggests that the intramolecular relaxation takes place within the time resolution of the system. In fact, the intramolecular relaxation in the $S_{2}$ state is considered to occur within $50 \mathrm{fs}$ in previous papers. ${ }^{23,24}$

Figure 6 shows time evolution of the fluorescence of $\beta$-carotene measured by the OKG method. Closed circles, triangles, and squares indicate the observed data points at different detected wavelengths 480,520 , and $560 \mathrm{~nm}$, respectively. The instrumental response function obtained from

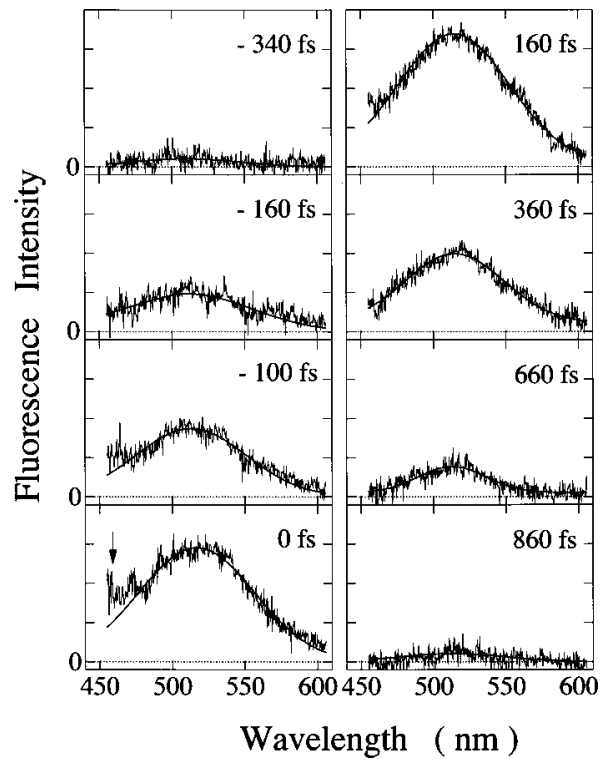

FIG. 5. Time-resolved fluorescence spectra of $\beta$-carotene in $n$-hexane solution for different delay times.

the time profile of the third order correlation function (Fig. 3) is also shown by a broken line. A solid curve is the best fit obtained by a single exponential decay of $210 \pm 20 \mathrm{fs}$ convoluted with the instrumental response function. The lifetime of the $S_{2}$ state was estimated to be $190-250$ fs by the transient absorption measurement ${ }^{22}$ and the up-conversion technique. ${ }^{23}$ The observed fluorescence decay time of $210 \mathrm{fs}$ is in good agreement with the estimated value from the previous measurements.

We clearly observed the time-resolved fluorescence spectra of $\beta$-carotene in femtosecond time regime, which has a very low fluorescence quantum yield and an ultrafast fluorescence decay time. This strongly shows that the OKG method is a powerful spectroscopic tool to investigate ultrafast carrier dynamics and relaxation processes of materials.

\section{SUMMARY}

Time-resolved luminescence spectroscopy by the OKG method has been developed to investigate ultrafast carrier

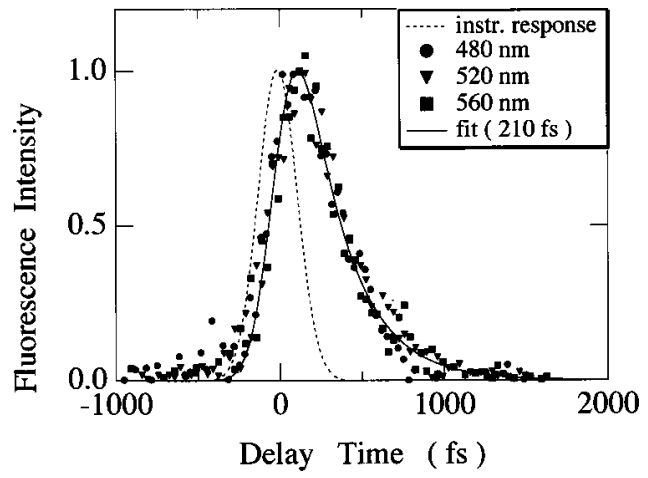

FIG. 6. Time evolution of fluorescence of $\beta$-carotene. Closed circles, triangles, and squares indicate the observed data points at different detected wavelengths 480,520 , and $560 \mathrm{~nm}$, respectively. The instrumental response function is also shown by a broken line. 
dynamics and relaxation processes of materials. The solid glasses with high nonlinear refractive indexes were used as the Kerr media to obtain a subpicosecond time resolution. The characteristics of our OKG method when we used a quartz plate as the Kerr medium are as follows: (1) the Kerr efficiency and the width of the instrumental response function are $5-10 \%$ and $\sim 250 \mathrm{fs}$, respectively-the time resolution of our OKG method becomes better than 100 fs after the convolution procedure, (2) tunability of the excitation wavelength covers a wavelength region of $380-420 \mathrm{~nm}$, and (3) observable spectral range of a time-resolved luminescence spectrum is $450-750 \mathrm{~nm}$.

The observable spectral range is limited by the wavelengths of excitation $(400 \mathrm{~nm})$ and gating laser pulses $(800$ $\mathrm{nm})$. By using the third harmonic from a Ti:sapphire regenerative amplifier or the output from an optical parametric amplifier as the excitation pulse of samples, and the second harmonic as the gating pulse, the observable spectral range as well as the tunability of the excitation will be easily extended. Such improvements for our OKG method are now in progress.

Our OKG method was applied to measure the timeresolved fluorescence spectra of $\beta$-carotene which shows a low fluorescence quantum yield and an ultrafast fluorescence decay time. The lifetime of the $S_{2}$ state was determined to be 210 fs by the OKG method.

The advantage of the OKG method relative to the conventional up-conversion technique is in its ability to directly obtain time-resolved luminescence spectra, which include important and fruitful information on carrier dynamics and relaxation processes of materials. For this advantage, we believe that the OKG method might replace the up-conversion technique to investigate the ultrafast carrier dynamics and relaxation processes of materials.

\section{ACKNOWLEDGMENTS}

This work was performed by using a femtosecond regenerative amplifier system at Instrumental Analysis Center of Yokohama National University. This work was partly supported by Grant-in-Aid for Scientific Research (B) (No. 11440091) and by Grant-in-Aid on priority areas, "Photoinduced Phase Transition and Their Dynamics, from the Ministry of Education, Science Sports and Culture of Japan." The Kurata Foundation and CASIO Science Promotion Foundation to J.T. and the Sasakawa Scientific Research Grant from The Japan Science Society to K.N. are also gratefully acknowledged.
${ }^{1}$ D. Block, J. Shah, and A. C. Gossard, Solid State Commun. 59, 527 (1986).

${ }^{2}$ J. Shah, IEEE J. Quantum Electron. 24, 276 (1988).

${ }^{3}$ J. Shah, T. C. Damen, B. Deveaud, and D. Block, Appl. Phys. Lett. 50, 1307 (1987).

${ }^{4}$ S. Tomimoto, H. Nansei, S. Saito, T. Suemoto, J. Takeda, and S. Kurita, Phys. Rev. Lett. 81, 417 (1998).

${ }^{5}$ S. Tomimoto, S. Saito, T. Suemoto, K. Sakata, J. Takeda, and S. Kurita, Phys. Rev. B 60, 7961 (1999).

${ }^{6}$ S. Iwai, S. Murata, and M. Tachiya, J. Chem. Phys. 109, 5963 (1998).

${ }^{7}$ M. A. Duguay and J. M. Hansen, Opt. Commun. 1, 254 (1969).

${ }^{8}$ G. R. Fleming, Chemical Application of Ultrafast Spectroscopy (Oxford, New York, 1986), pp. 124-224.

${ }^{9}$ D. Hulin, J. Etchepare, A. Antonetti, L. L. Chase, G. Grillon, A. Migus, and A. Mysyrowicz, Appl. Phys. Lett. 45, 993 (1984).

${ }^{10}$ A. Brun, P. Georges, G. Le Saux, and F. Salin, J. Phys. D 24, 1225 (1991).

${ }^{11}$ H. S. Albrecht, P. Heist, J. Kleinschmidt, D. V. Lap, and T. Schröder, Appl. Opt. 32, 6659 (1993).

${ }^{12}$ H. S. Albrecht, P. Heist, J. Kleinschmidt, D. V. Lap, and T. Schröder, Appl. Phys. B: Photophys. Laser Chem. 55, 362
(1992).

${ }^{13}$ J. Takeda, K. Nakajima, S. Kurita, S. Tomimoto, S. Saito, and T. Suemoto, J. Lumin. 87-89, 927 (2000).

${ }^{14}$ M. Nagai, R. Shimano, and M. Kuwata-Gonokami, J. Lumin. 87-89, 1926 (2000).

${ }^{15}$ Y. Kanematsu, H. Ozawa, I. Tanaka, and S. Kinoshita, J. Lumin. 87-89, 917 (2000).

${ }^{16}$ R. Adair, L. L. Chase, and S. A. Payne, Phys. Rev. B 39, 3337 (1989).

${ }^{17}$ R. Righini, Science 262, 1386 (1993).

${ }^{18}$ B. S. Hudson, B. E. Kohler, and K. Schulten, Excited States 6, 1 (1982).

${ }^{19}$ B. S. Hudson and B. E. Kohler, Chem. Phys. Lett. 14, 299 (1972).

${ }^{20}$ T. Gillgro and R. J. Cogdell, Chem. Phys. Lett. 158, 312 (1989).

${ }^{21}$ S. L. Bondarev, S. M. Bachilo, S. S. Dvornikov, and S. A. Tikhomirov, J. Photochem. Photobiol., A 46, 315 (1989).

${ }^{22}$ A. P. Shreve, J. L. Trautman, T. G. Owens, and A. C. Albrecht, Chem. Phys. Lett. 178, 89 (1991).

${ }^{23}$ H. Kandori, H. Sasabe, and M. Mimuro, J. Am. Chem. Soc. 116, 2671 (1994).

${ }^{24}$ J. Watanabe, H. Takahashi, J. Nakahara, and T. Kushida, Chem. Phys. Lett. 213, 351 (1993). 